\title{
Early patellofemoral articular cartilage degeneration in a rat model of patellar instability is associated with activation of the NF-KB signaling pathway
}

Wei Lin, Huijun Kang, Yike Dai, Yingzhen Niu, Guangmin Yang, Jinghui Niu, Ming Li and Fei Wang*

\begin{abstract}
Background: Patellar instability (PI) often increases the possibility of lateral patellar dislocation and early osteoarthritis. The molecular mechanism of early articular cartilage degeneration during patellofemoral osteoarthritis (PFOA) still requires further investigation. However, it is known that the NF-kB signaling pathway plays an important role in articular cartilage degeneration. The aim of this study was to investigate the relationship between the NF-KB signaling pathway and patellofemoral joint cartilage degeneration.

Methods: We established a rat model of PI-induced PFOA. Female 4-week-old Sprague-Dawley rats $(n=120)$ were randomly divided into two groups: the PI $(n=60)$ and control group $(n=60)$. The distal femurs of the PI and control group were isolated and compared 4, 8, and 12 weeks after surgery. The morphological structure of the trochlear cartilage and subchondral bone were evaluated by micro-computed tomography and histology. The expression of NF-KB, matrix metalloproteinase (MMP)-13, collagen X, and TNF-a were evaluated by immunohistochemistry and quantitative polymerase chain reaction.

Results: In the PI group, subchondral bone loss and cartilage degeneration were found 4 weeks after surgery. Compared with the control group, the protein and mRNA expression of NF-KB and TNF-a were significantly increased 4, 8, and 12 weeks after surgery in the PI group. In addition, the markers of cartilage degeneration MMP13 and collagen $\mathrm{X}$ were more highly expressed in the PI group compared with the control group at different time points after surgery.

Conclusions: This study has demonstrated that early patellofemoral joint cartilage degeneration can be caused by $\mathrm{PI}$ in growing rats, accompanied by significant subchondral bone loss and cartilage degeneration. In addition, the degeneration of articular cartilage may be associated with the activation of the NF-kB signaling pathway and can deteriorate with time as a result of PI.
\end{abstract}

Keywords: Patellar instability, Patellofemoral osteoarthritis, NF-kB signal pathway

\footnotetext{
* Correspondence: doctorlinw@163.com

Department of Orthopedic Surgery, Third Hospital of Hebei Medical

University, No. 139 Ziqiang Road, 050051 Shijiazhuang, Hebei, China
}

(c) The Author(s). 2021 Open Access This article is licensed under a Creative Commons Attribution 4.0 International License, which permits use, sharing, adaptation, distribution and reproduction in any medium or format, as long as you give appropriate credit to the original author(s) and the source, provide a link to the Creative Commons licence, and indicate if changes were made. The images or other third party material in this article are included in the article's Creative Commons licence, unless indicated otherwise in a credit line to the material. If material is not included in the article's Creative Commons licence and your intended use is not permitted by statutory regulation or exceeds the permitted use, you will need to obtain permission directly from the copyright holder. To view a copy of this licence, visit http://creativecommons.org/licenses/by/4.0/ The Creative Commons Public Domain Dedication waiver (http://creativecommons.org/publicdomain/zero/1.0/) applies to the data made available in this article, unless otherwise stated in a credit line to the data. 


\section{Background}

Osteoarthritis (OA) is a chronic joint disease that affects more than 100 million people worldwide [1, 2]. Usually, OA most strongly affects the knee joint, a threecompartment structure that includes the patellofemoral joint (PFJ) and the medial and lateral tibiofemoral joints. Previous studies on knee OA have focused on the tibiofemoral joints, and little research has been done on the PFJ [2]. However, isolated patellofemoral osteoarthritis (PFOA) is not only common but has a high prevalence in men (18.5-19.0\%) and women (17.1-34.0\%) aged $55-60$ years $[3,4]$. Furthermore, PFOA is the main cause of anterior knee pain and disability [5].

The PFJ is a unique and complex structure [6], which is stabilized by a complex multivariate relationship of osseous joint geometry and force vectors generated by the capsuloligamentous stabilizers and quadriceps femoris [7]. Patellar instability (PI) is frequently caused by pathological changes involving PFJ stabilizers, which increase the likelihood of lateral patellar dislocation and early OA [8]. Additionally, PI is related to trochlear dysplasia [8]. Schottle et al. demonstrated that trochlear dysplasia of the femur is a predisposing factor for recurrent PI [9]. Huri also demonstrated that PI is related to trochlear dysplasia [10]. In the general population, the incidence of primary PI is 5.8 per 100,000 , with a higher incidence in more active and younger individuals [11]. Some risk factors, such as lateralization of tibial tuberosity, trochlear dysplasia, and the medial patellofemoral ligament, are believed to promote PI [12]. Biomechanical investigations have suggested that the medial patellofemoral ligament plays a crucial role in restraining lateral patellar translation and provides $50-60 \%$ of PI; the retinacular fibers and patellomeniscal ligament were also demonstrated to be significant medial stabilizers. It is speculated that these ligament structures can provide proprioceptive signals for surrounding muscle tissues in addition to their biomechanical properties [13]. Although the PFJ plays an important role in knee OA, studies on the pathogenesis of PFOA are scarce [14]. The spontaneous PFOA model is characterized by slow disease progression, long study duration, and frequent changes in outcome [15]. A rat model of joint inflammation consists of intra-articular injection of monosodium iodoacetate that chemically induces chondrocyte death, but may not be a representative model of OA $[16,17]$. The surgically induced OA model is mainly used to study tibiofemoral osteoarthritis, with only few studies on PFOA [18-20]. Additionally, the influence of PI on PFOA remains unclear.

It is well known that collagen $\mathrm{X}[21,22]$ and MMP-13 [23], markers of cartilage degeneration, lead to chondrocyte imbalance and matrix degradation. Compared with normal cartilage, the cartilage of OA patients exhibits up-regulated TNF-a levels [24]. Recently, the NF-kB signaling pathway was found to play an important role in regulating inflammatory mediators associated with $\mathrm{OA}$ [25]. This pathway serves as a bridge between cartilage degeneration and development, and few studies have investigated the role of PFOA in early cartilage degeneration.

Therefore, we investigated the relationship between cartilage degeneration during PFOA and the NF- $\mathrm{kB}$ signaling pathway by employing immunohistochemistry to assess the histological characteristics of cartilage and quantitative real-time polymerase chain reaction (qRT$\mathrm{PCR})$ to assess the different expressions of $\mathrm{NF}-\mathrm{\kappa B}$, MMP-13, collagen X, and TNF-a in cartilage at different time points in a growing rat model of PI.

\section{Methods}

\section{Study design}

This study was approved by the Animal Ethics Committee of the Third Hospital of Hebei Medical University (approval number: Z2019-005-1). Female 4-week-old Sprague-Dawley rats (50-80 g) were provided by the Laboratory Animal Center of Hebei Medical University. Rats ( $n=120)$ were randomly divided into two groups: the control $(n=60)$ and PI group $(n=60)$. In the control group, the rats' left knees did not undergo any surgery. In the PI group, the rats' left knees underwent surgery to induce PI. Afterwards, left distal femur tissues were collected $(n=20$ knees/time point in each group) (Fig. 1). All the rats were sacrificed by an overdose of anesthesia at 4, 8, and 12 weeks after surgery. According to animal euthanasia guidelines [26, 27], rats were intraperitoneally injected with an overdose of pentobarbital sodium $(200 \mathrm{mg} / \mathrm{kg})$. Rats were placed in a controlled environment $(60 \pm 5 \%$ humidity, $12 / 12 \mathrm{~h}$ light/dark cycle, $22 \pm 3{ }^{\circ} \mathrm{C}$ ) and were free to obtain food and water. The animals were accustomed to the laboratory environment with an adaptation period of 1 week before experimentation.

\section{Surgical technique}

Before surgery, pentobarbital sodium $(30 \mathrm{mg} / \mathrm{kg}$, intraperitoneal injection) was used to induce anesthesia in all rats; the left knee was placed in an extended state. After the surgical area was shaved, the surgery was prepared aseptically. A midline skin incision was performed; after the skin and subcutaneous tissue were separated, the capsule was exposed through the medial approach of the knee. Similar methods of surgery-induced PI have been reported in our previous studies [28, 29]. In this study, the medial retinaculum of the knee joint was cut off, and PI could be observed during surgery. The incision was then closed without reconstruction of the medial patellofemoral ligament, retinaculum, or capsules. After surgery, dressing was applied (Fig. 2). For post-operative 


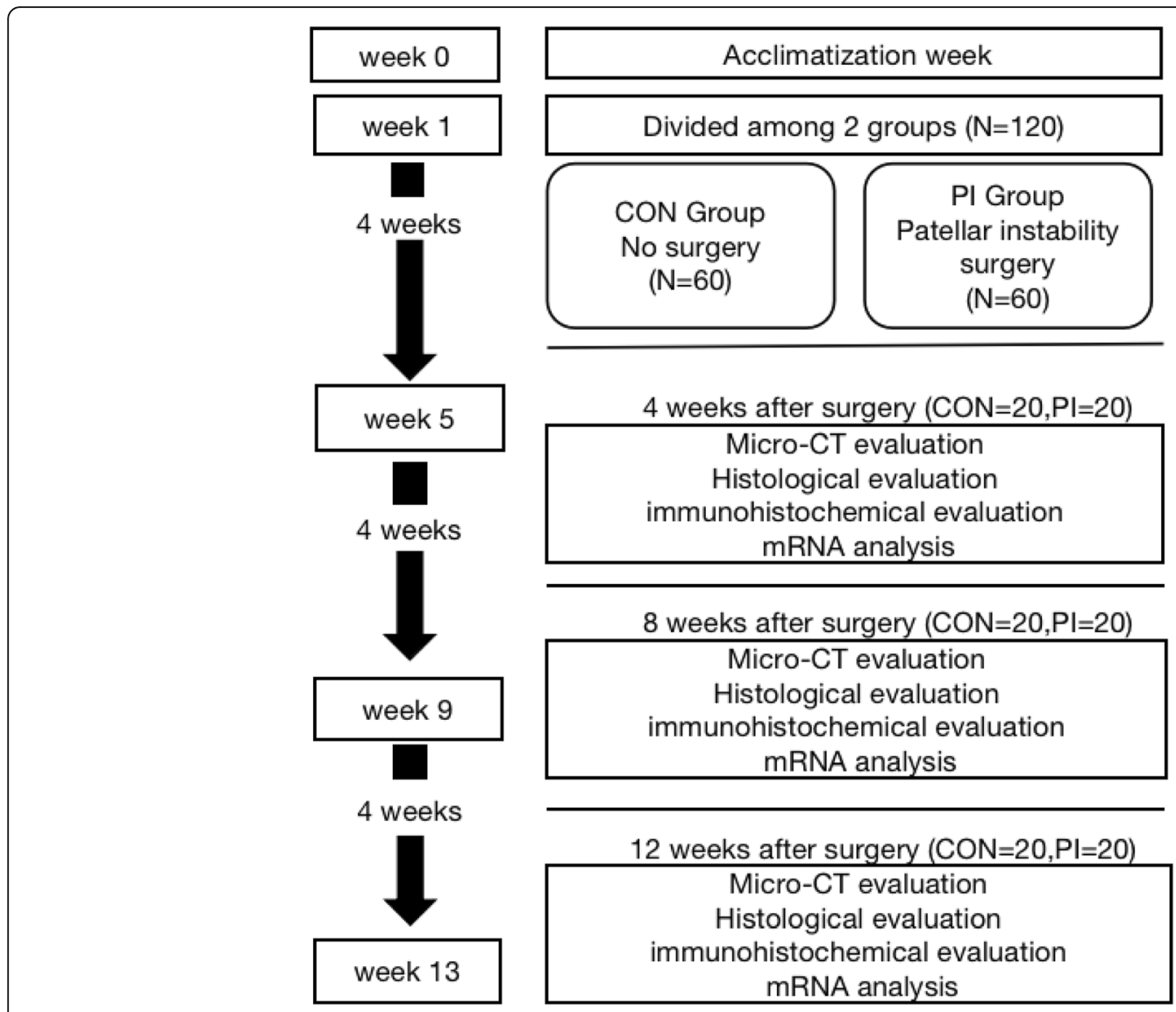

Fig. 1 Schematic of the experimental protocol

care, animals were provided with $24 \mathrm{~h}$ quiet recovery time in a warm and dry environment. They were also provided with food to promote gastrointestinal peristalsis and to prevent stagnation after fully awakening. To control post-operative pain, rats were administered acetaminophen $(30 \mathrm{mg} / \mathrm{kg}$, daily) for 5 days.

\section{Micro-computed tomography (micro-CT) assessment}

In order to assess PI-induced changes in the subchondral bone microstructure, the bone tissues of distal femurs were carefully harvested and scanned via a microCT (SkyScan model 1076, Skyscan, Belgium) at 4, 8, and 12 weeks after surgery $(n=5$ knees/time point in each
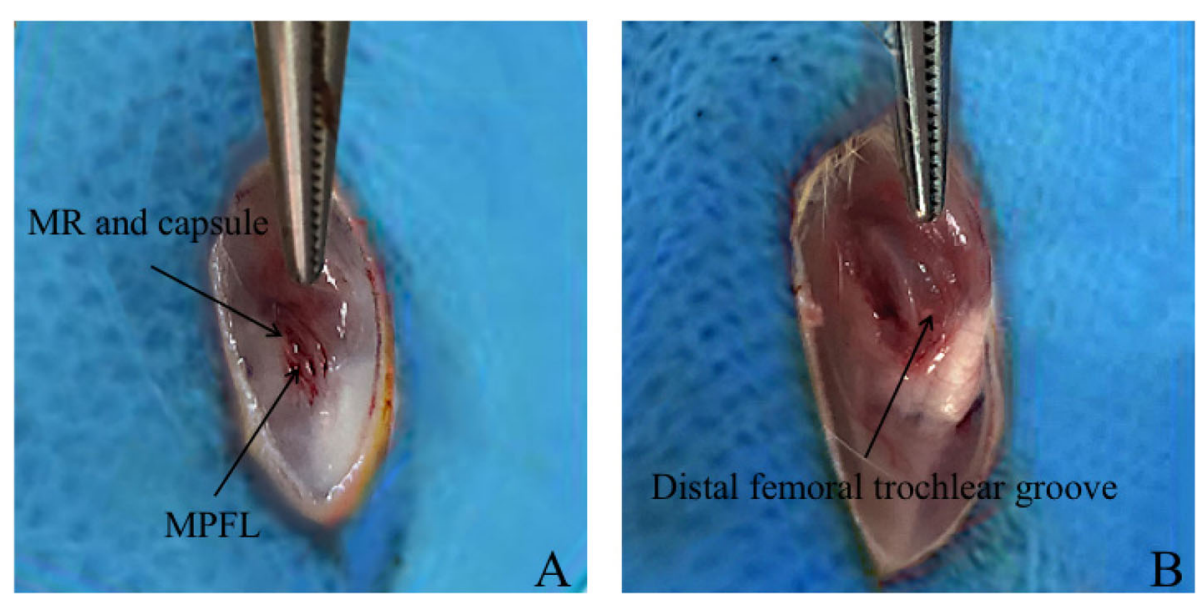

Fig. 2 Detailed procedure of the operations: a, Cut off the medial soft tissue restraints (MPFL: medial patellofemoral ligament, MR: medial retinaculum); b, Postoperative PI (Patellar instability). Distal femoral groove can be noticed after the release of medial soft tissue restrains 
group). All scans were performed with a voxel size of $10 \mu \mathrm{m}$ at $50 \mathrm{kV}$ and $800 \mu \mathrm{A}$. The region of interest (ROI) was located transversely below the trochlea with a green rectangle $(4 \times 3 \mathrm{~mm})$ (Fig. 3). Based on previous studies, the following representative parameters were selected to assess changes in subchondral bone [2]: bone volume to total volume fraction (BV/TV fraction), bone mineral density (BMD), trabecular (TB) thickness, TB separation (SP), structure model index (SMI), TB pattern factor (PF), TB number, and degree of anisotropy (DA).

\section{Histology}

The bone tissues of distal femurs were carefully harvested at each time point $(\mathrm{n}=5 \mathrm{knees} /$ time point in each group), fixed with $4 \%$ polyformaldehyde, decalcified in $10 \%$ EDTA until completely demineralized, and embedded in paraffin. Sections $(5 \mu \mathrm{m})$ were cut along the femoral axis to obtain the transverse images of the trochlea sulcus. Safranin O was used to evaluate cartilage glycosaminoglycans. Cartilage degradation was evaluated by subjecting protein to fast green counter staining and scored using a semi-quantitative histopathological grading system based on the Osteoarthritis Research Society International (OARSI) score [30, 31]. After observation with a microscope, a camera was used to acquire images of the representative sections. Chondrocyte numbers were quantified in the superficial and middle zone and expressed as numbers $/ \mathrm{mm}^{2}$. Values were calculated from at least five nonconsecutive sections per region.

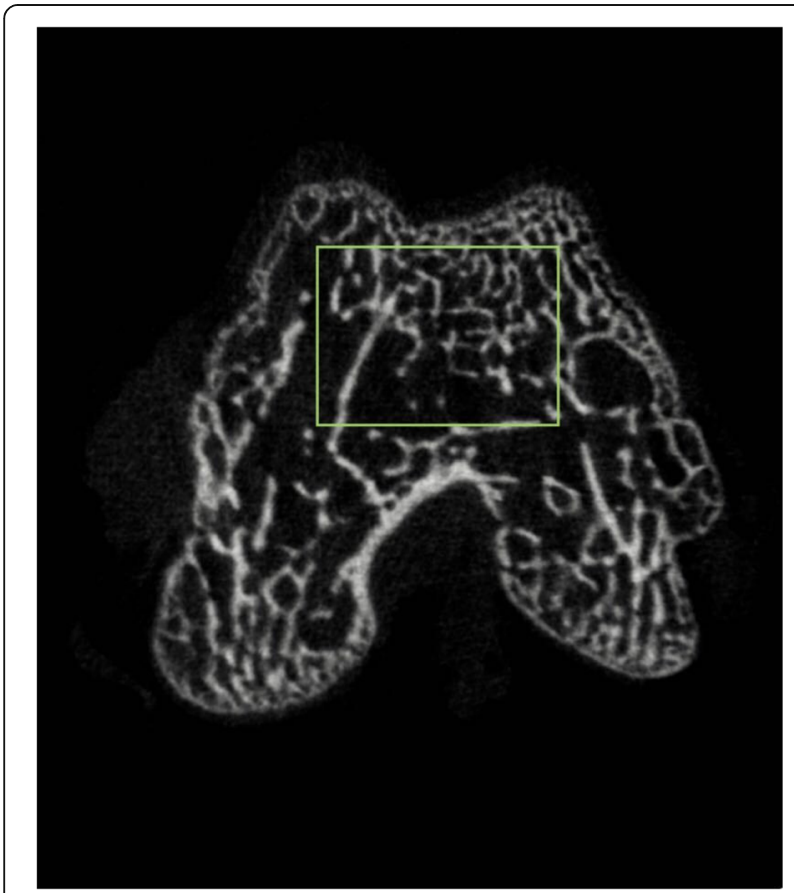

Fig. 3 Axial view of the distal femur. ROI was shown within the green rectangular area. (ROl: region of interest)

\section{Immunohistochemistry}

The slices were deparaffinized in xylene, rehydrated, and washed three times for $5 \mathrm{~min}$ with phosphate buffered saline at room temperature. Endogenous peroxidase activity was blocked by $3 \%$ hydrogen peroxide $\left(\mathrm{H}_{2} \mathrm{O}_{2}\right)$ for $10 \mathrm{~min}$. Antigen retrieval was performed by microwave treatment in $10 \mathrm{~mm}$ of sodium citrate (pH 6.0) for $10 \mathrm{~min}$. This was followed by overnight incubation at $4{ }^{\circ} \mathrm{C}$ with anticollagen X (BoAoSen, BeiJing, China), anti-MMP-13 (Servicebio, WuHan, China), anti-TNF-a (ABclonal, WuHan, China), and anti-NFKP65 (Servicebio, Wu Han, China) at a dilution of 1:50. The primary antibody was omitted from the negative control reaction. Afterwards, images were acquired with a $20 \times 100$ objective lens from five randomly selected areas from each slice in each group ( $\mathrm{n}=5$ knees/ time point in each group). The tissue filled the entire field of view, and the background light of each photo was the same. Image-Pro Plus 6.0 software (Media Cybernetics, Rockville, MD, USA) was used for image analysis and choose the same brown color as the uniform standard for judging all positive photos. Data regarding integrated optic density of positive cell and the pixel area of tissue were acquired for all images. The areal density was defined as the integrated optic density divided by the pixel area of tissue and was positively correlated with positive cell expression.

\section{qRT-PCR}

At 4,8 , and 12 weeks after surgery, the cartilage was peeled off from the trochlear with a blade and was analyzed by qRT-PCR for mRNA expression ( $\mathrm{n}=5$ knees/ time point in each group). Trizol reagent (Servicebio, Wuhan, China) was used to extract RNA from the cartilage. mRNA was translated into cDNA by the RevertAid ${ }^{\text {me }}$ first strand cDNA synthesis kit (Thermo, \#K1622) according to manufacturer's instructions. The target genes were MMP-13, collagen X, TNF- $\mathrm{a}$, and NF- $\mathrm{kB}$ p65; all genes were analyzed by applying the sequence detection system, and the expression of related mRNA was standardized relative to the GAPDH gene and calculated with the $2^{-\Delta \Delta C t}$ (cycle threshold) method. The primers used are shown in Table 1. We performed each experiment three times and obtained the average values.

\section{Statistical analysis}

All data values were expressed as mean \pm standard deviation. Data were analyzed with the statistical software GraphPad Prism (GraphPad software V7, La Jolla, CA, USA).The Shapiro-Wilk test was used to evaluate normality, the Levene's test was used to evaluate the homogeneity of variance, and two-way analysis of variance was used to evaluate the parametric data between the two groups at each time point. $p<0.05$ was considered statistically significant. According to the results of the preliminary experiments, each group and each time 
Table 1 Primers used for amplification of target genes and GAPDH

\begin{tabular}{lll}
\hline & Forward primer sequence & Reverse primer sequence \\
\hline Collagen $X$ & CCCTTCTGCTGCTAGTGTCCT & GGAATGCCTTGTTCTCCTCTTAC \\
MMP-13 & TGCATACGAGCATCCATCCC & CGTGTCCTCAAAGTGAACCGC \\
TNF-a & CCACCACGCTCTTCTGTCTACTG & TGGGCTACGGGCTTGTCACT \\
NF-KB p65 & CTTCACTCGGAGACTGGAACCT & AAATCCTTCCCAAACTCCACC \\
GAPDH & CTGGAGAAACCTGCCAAGTATG & GGTGGAAGAATGGGAGTTGCT \\
\hline
\end{tabular}

point required at least six rats, with a confidence of $90 \%$ and an efficacy of $80 \%(1-\beta)$ [32].

\section{Results}

\section{Micro-CT measurements of subchondral bone}

In the PI group, significant bone loss was observed at 4 weeks compared with the time-matched controls; the loss of bone mass gradually increased, and the subchondral bone plate became obviously thinner (Fig. 4). Micro-CT revealed that the BV/TV fraction decreased $(p<0.05)$, while the SMI and TB SP increased 4 weeks after surgery $(p<0.05)$ (Fig. 4$)$. Compared with the time-matched controls, TB thickness, TB number, and BMD decreased $(p<0.05)$, while the TB PF increased 8 and 12 weeks after surgery $(p<$ 0.05) (Fig. 4).
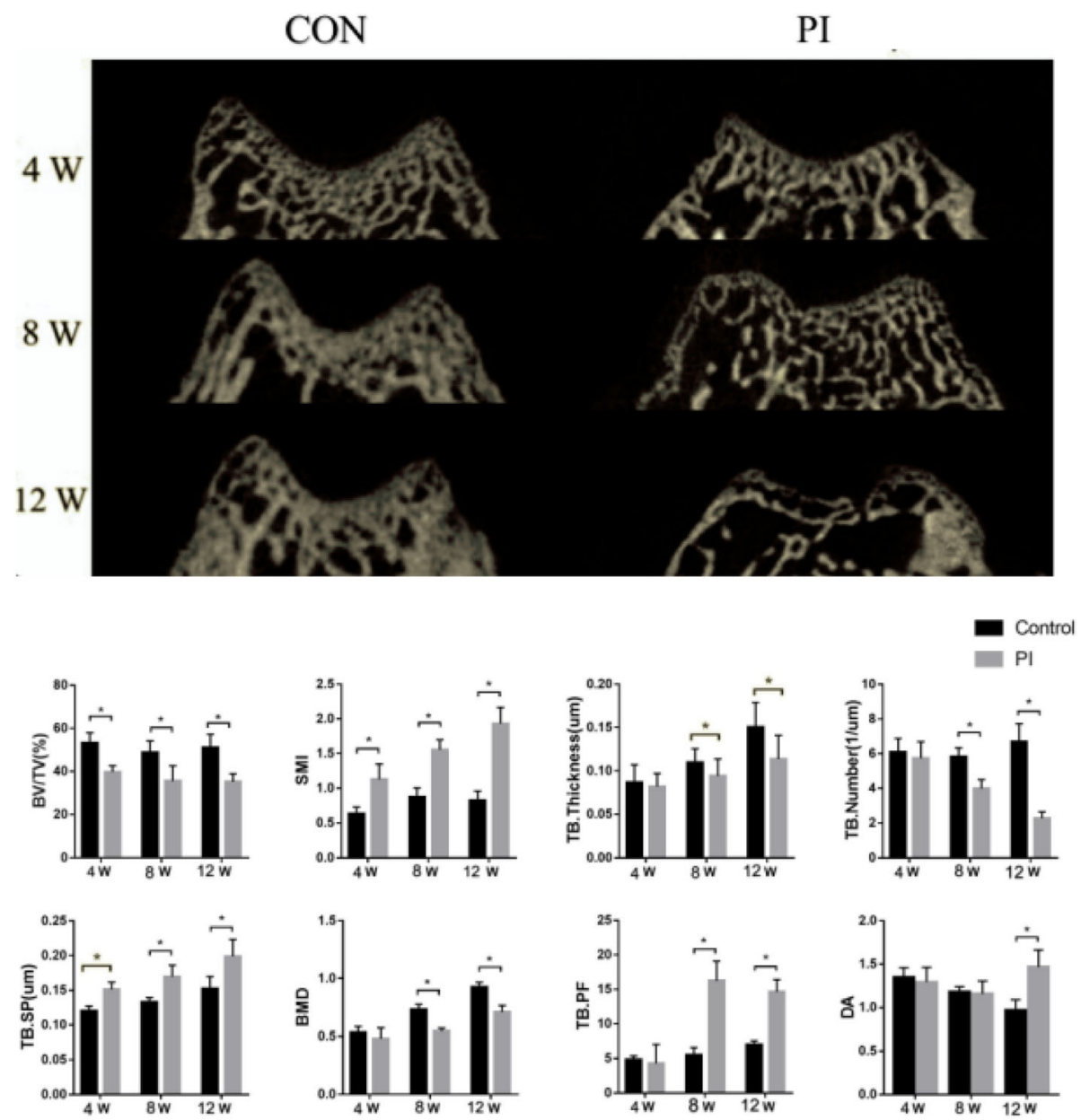

Fig. 4 Micro-CT showed the configuration of femoral trochlear and the subchondral bone in the CON and PI groups at all times points. In the PI group, the center of the trochlear groove become flat, significantly bone loss was observed at 8,12 weeks after surgery, and the loss of bone mass gradually aggravated. Architectural parameters of subchondral bone samples of different groups. ${ }^{*} P<0.05$ (BV/TV: bone volume to total volume; BMD: bone mineral density; TB: trabecular; SP: separation; SMI: structure model index; PF: pattern factor; DA: degree of anisotropy; CON Group, control group; PI Group, patellar instability group) 


\section{PI leads to articular cartilage degeneration}

Four weeks after surgery, we determined the relative positive staining area and the structural changes of the cartilage surface layer. We observed cell aggregation, loss of cartilage structure, and the Safranin O staining reduced significantly consistent with OA, 8 and 12 weeks after surgery (Fig. 5). In the PI group, safranin $\mathrm{O}$ and fast green histochemical staining showed that the cartilage surface was eroded and irregular and that the number of chondrocytes in the superficial and middle zone was significantly reduced $(p<0.05) 12$ weeks after surgery (Fig. 5). However, in the control group, the chondrocytes were flattened and the cartilage surface was smooth at different time points. Compared with the control group, higher OARSI scores were observed 4,8 , and 12 weeks after surgery in the PI group $(p<0.05)$ (Fig. 5).
PI increased the expression of MMP-13, collagen X, TNF- $a$, and NF-KB P65

Immunohistochemical analysis revealed that there were significant differences in MMP-13, collagen X, TNF-a, and NF- $\mathrm{kB}$ p65 4, 8, and 12 weeks after surgery $(p<$ 0.05) (Fig. 6). Furthermore, qRT-PCR analysis revealed expression patterns similar to those of the immunohistochemical analysis $(p<0.05)$ (Fig. 7).

\section{Discussion}

In this study, we successfully established a rat PI-induced PFOA model and found that PI aggravated subchondral bone loss and cartilage degeneration, which worsened over time. Furthermore, we observed significantly elevated NF$\mathrm{\kappa B}$ protein and mRNA levels in the PFOA model, which indicates that PI-induced cartilage degeneration may be associated with activation of the NF- $\mathrm{kB}$ signaling pathway.

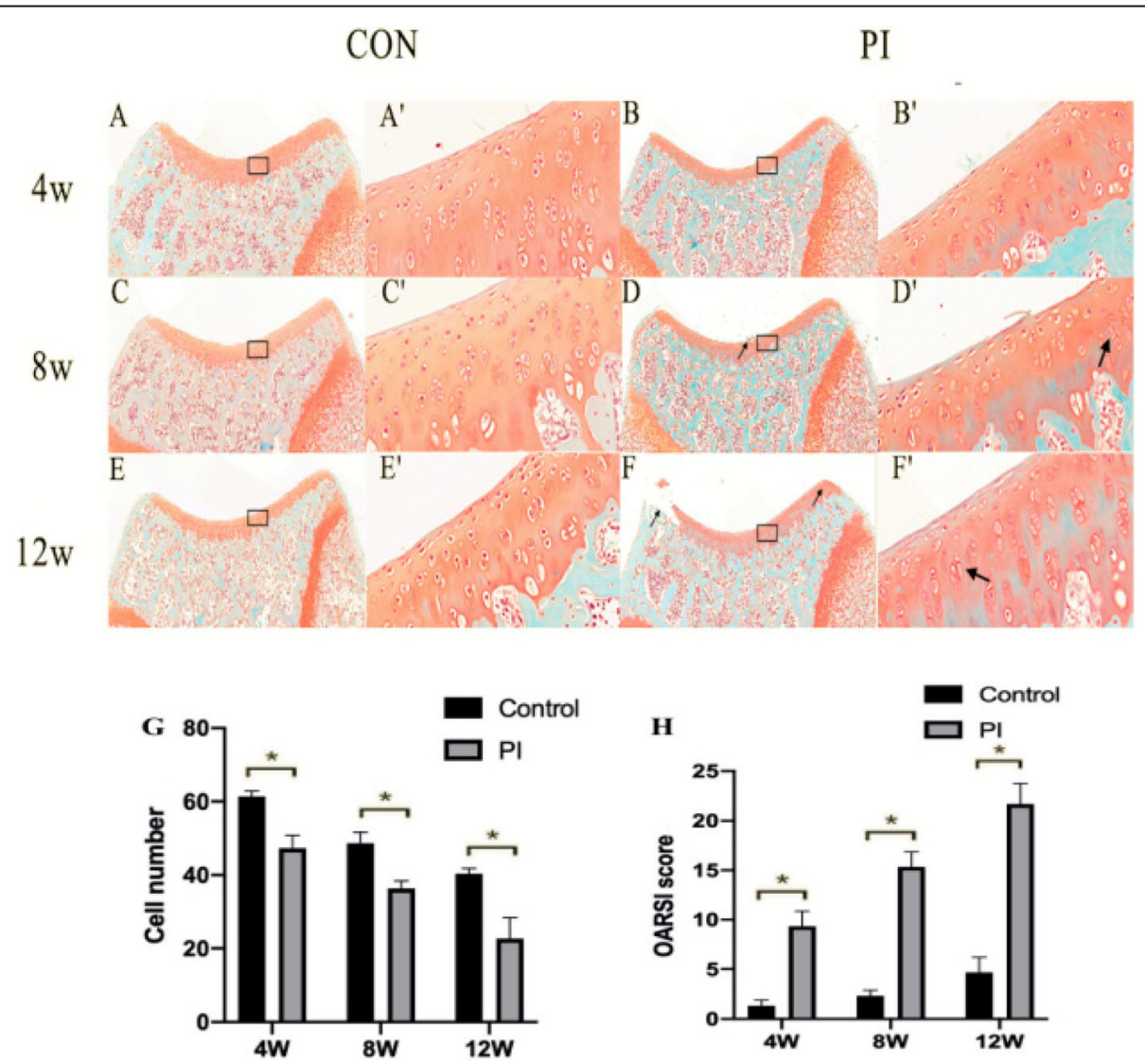

Fig. 5 SafraninO and fast green staining in cartilage of rats. CON and PI group respectively: a, b 4 weeks; c, d 8 weeks; and (e, f) 12 weeks. (A'-F') images at a higher magnification of boxed areas in $(\mathbf{a}-\mathbf{f})$. In the PI group, safranin $\mathrm{O}$ and fast green histochemical staining showed that the surface of the cartilage was irregular and destroyed at 8, 12 weeks after surgery(the thin black arrow in $\mathbf{d}_{1} \mathbf{f}$ ). In $\mathbf{D}^{\prime}, \mathbf{F}^{\prime}$, we observed cell aggregation(the thick black arrow), and the number of chondrocytes in the superficial and middle zone was significantly reduced, which was consistent with OA. $\mathbf{g}$ estimating cell numbers of the superficial and middle zone; $\mathbf{h}$ OARSI score for articular cartilage: increased OARSI score and decreased cell number were observed in the PI Group at 4,8, and 12 weeks after surgery. ${ }^{*} p<0.05$ (CON Group, control group; PI Group, patellar instability group) 


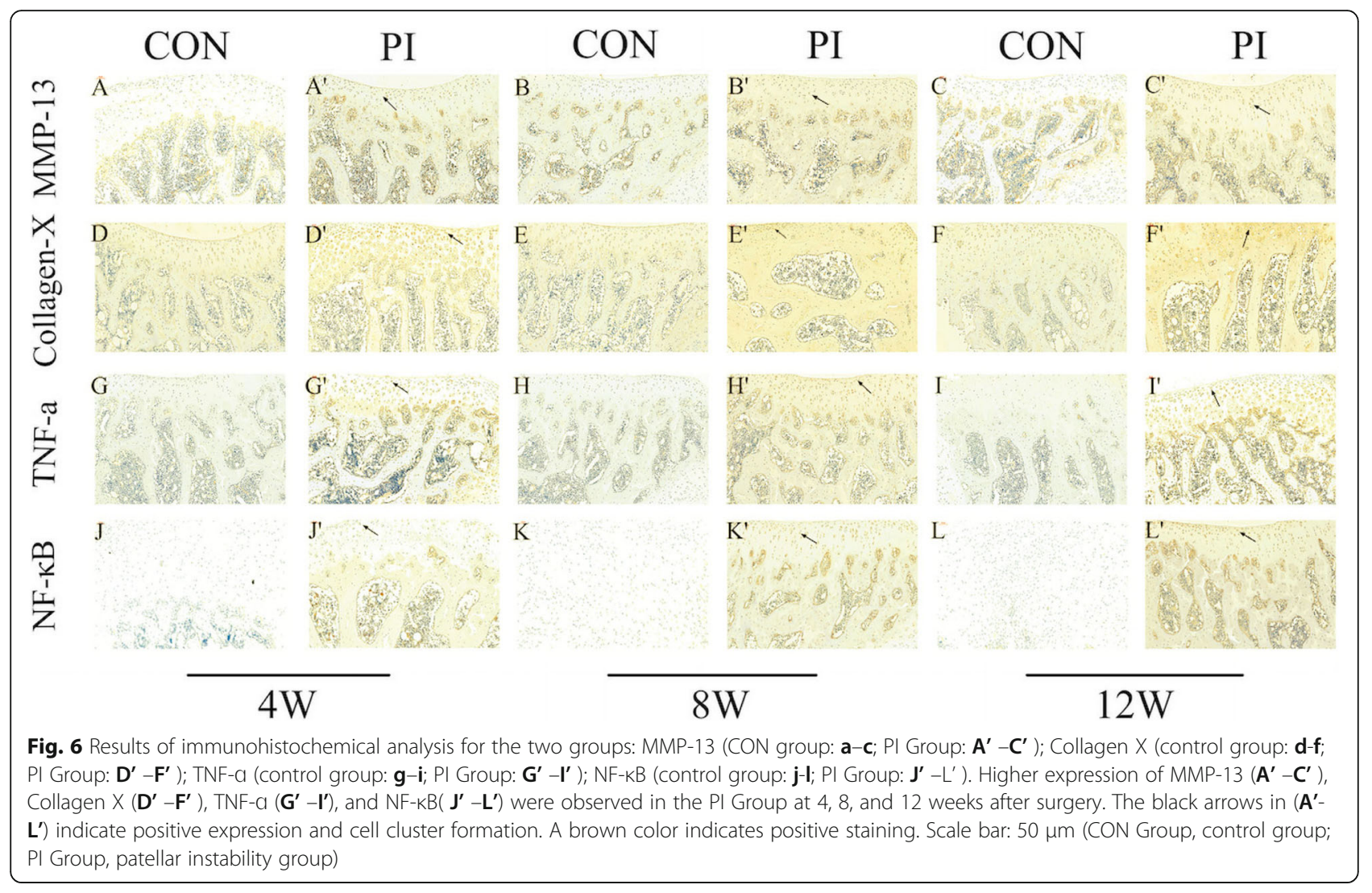

The present PFOA rat model exhibited obvious cartilage damage, which might be related to PI-induced patellofemoral hypertension and an abnormal load of PFOArelated structural damage [33]. One study demonstrated that mechanical stress is an important factor affecting cartilage and bone development [34]. PI-induced mechanical stress can destroy the integrity and dynamic balance of the PFJ. With the development of PI, the load in the PFJ redistributes, and the microstructure of the trabecular bone changes. The most obvious change was observed in the distal femur; the BV/TV fraction, TB thickness, TB number, and BMD decreased with time after surgery. This may be related to the abnormal pressure load that increase osteoclast activity, which may lead to a significant increase in osteoclast bone absorption in subchondral bone [35]. Furthermore, cartilage degradation was apparent from 4 weeks after surgery. In the PI group, we observed cell aggregation, loss of cartilage structure and the Safranin $\mathrm{O}$ staining reduced significantly at 8 and 12 weeks after surgery, this is consistent with human $\mathrm{OA}$. The safranin $\mathrm{O}$ and fast green histochemical staining showed that the cartilage surface was eroded and irregular, the number of chondrocytes in the superficial and middle zone was significantly reduced at 12 weeks after surgery. However, in the control group, the chondrocytes were flattened and the cartilage surface was smooth at different time points.
These results suggest that the changes in the microstructure and morphology of subchondral bone may be accompanied by gradual cartilage degradation and may further aggravate cartilage degradation during the process of PFOA [29].

The main feature of OA affecting the joint is the degeneration of articular cartilage. And, the altered phenotype of articular chondrocytes is the initial step in the progression of $\mathrm{OA}$ disease [36, 37]. In patients with osteoarthritis, MMP-13 is the main member of MMP family expressed in cartilage, but not in normal adults [38-40]. MMP-13 levels in patients with osteoarthritis indicate the degree of cartilage degeneration [38-40]. Activated MMPs catalyze the decomposition of cartilage matrix and induces chondrocyte apoptosis, leading to cartilage damage [41]. It has been found that MMP-13 can degrade collagen II in human OA [42]. Additionally, the reduced load decreases the proteoglycan content and causes cartilage thinning, which leads to cartilage degeneration [43]. Collagen $\mathrm{X}$ is a cartilage-specific collagen. Under normal circumstances, it is limited to the hypertrophic area of the growth plate, where it participates in endochondral ossification. Although Collagen $\mathrm{X}$ is not a component of normal articular cartilage, it is present in OA cartilage, especially in the deep regions where hypertrophic chondrocyte clusters are observed. Mutations in the Collagen X gene (COL10A1) lead to various forms 

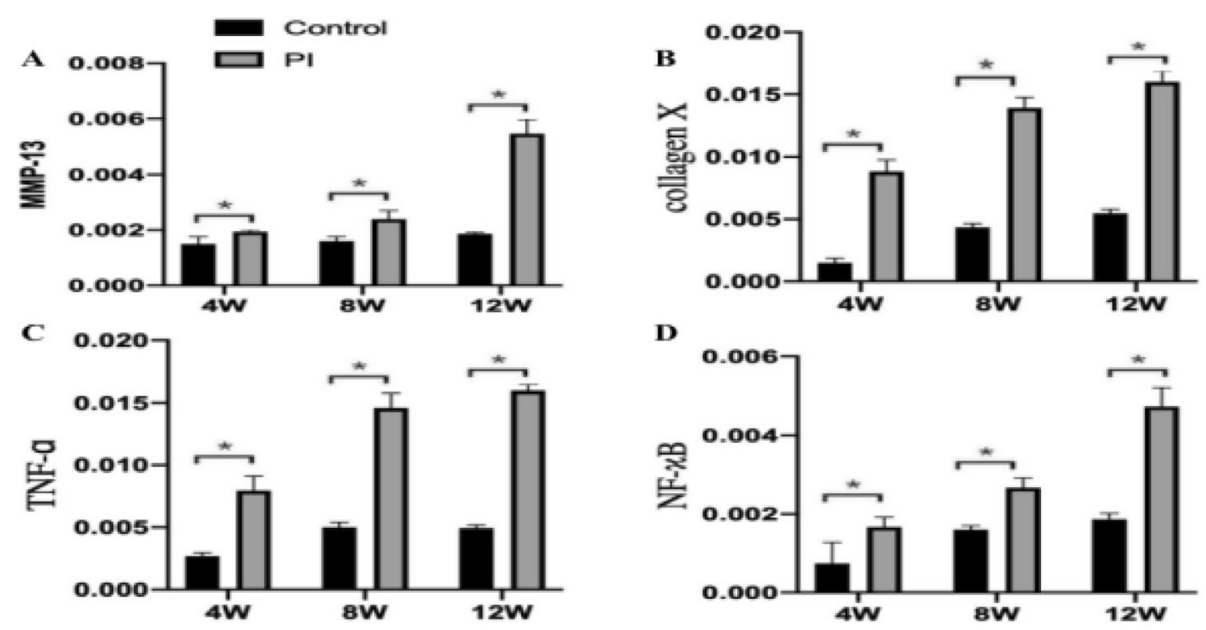

D
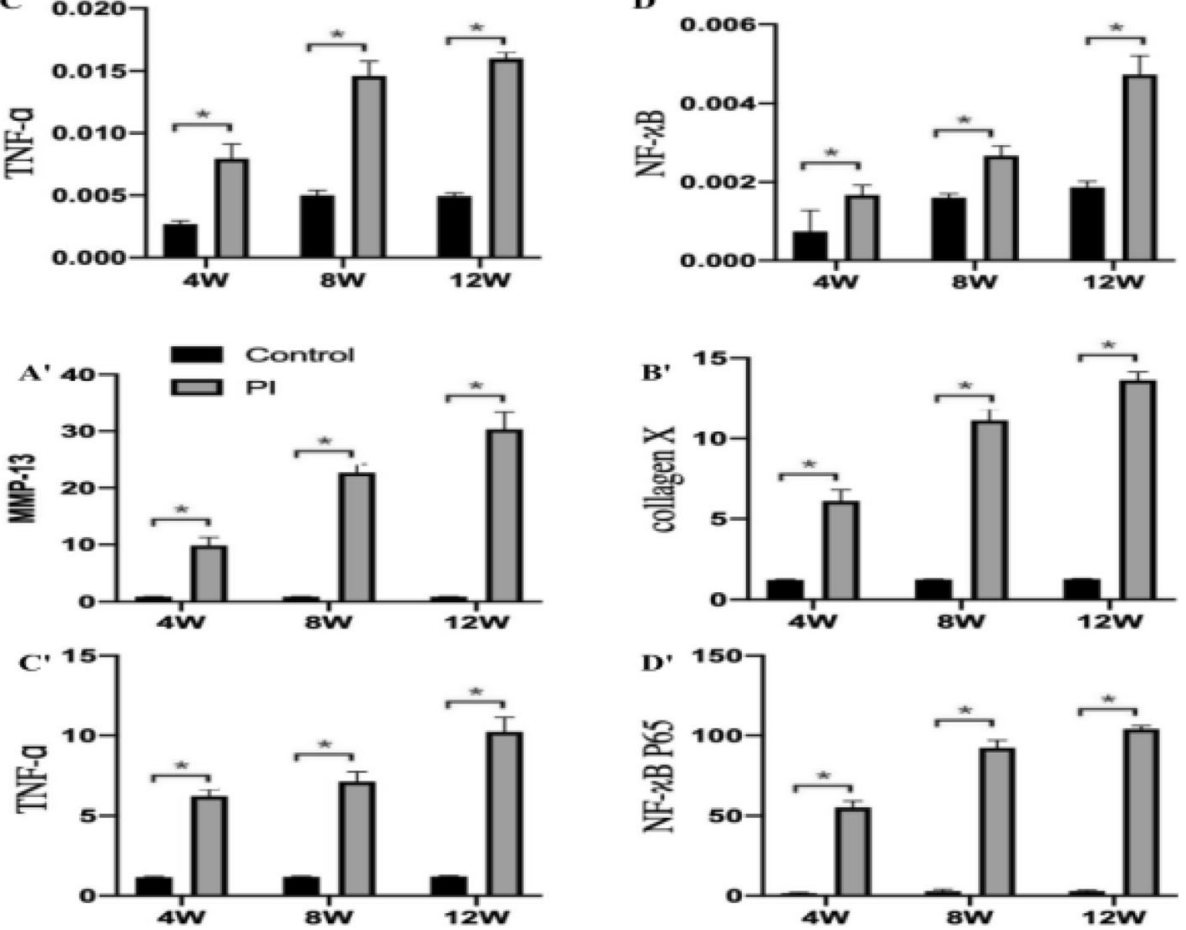

Fig. 7 The areal density of immunoistochemical analyses (a-d) and target gene expression of qRT-PCR evaluation ( $\mathbf{A}^{\prime}-\mathbf{D}^{\prime}$ ) in the two groups: showed higher expression of MMP-13, Collagen X, TNF-a, and NF-kB in the PI Group at 4, 8, and 12 weeks after surgery. ${ }^{*} p<0.05$ (PI Group, patellar instability group)

of metaphyseal dysplasia [44]. These studies are consistent with our findings. In the present study,we found that the expression of MMP-13 and Collagen X in immunohistochemistry and RT-PCR level were significantly higher. We believe that PI can significantly reduce the pressure in the PFJ, resulting in cartilage degeneration. In conclusion, our findings support the view that articular chondrocytes of the PI model exhibit the same molecular phenotype as the early stage of OA.

The pathogenesis of OA is not completely clear; however, it is closely related to many inflammatory factors [45]. It has been confirmed that the levels of proinflammatory cytokines, such as TNF-a and IL-1b, increase sharply during the early stages of OA progression. Animal and in vitro studies have confirmed that the inflammatory cytokine TNF-a can induce bone resorption and enhance cartilage degradation in OA $[46,47]$. In our study, we found higher immunohistochemical and mRNA expression of TNF-a, which can stimulate the production of other cytokines, such as IL-6, prostaglandin, and MMP [46] as well as decrease type II collagen and proteoglycan levels [48]. This may cause PFJ cartilage degradation after PI.

It is generally believed that the NF- $\mathrm{KB}$ signaling pathway plays a significant role in regulating inflammatory mediators associated with OA pathogenesis [25]. NF-kB most likely combines with the inhibitory subunit ІкB $\alpha$ and presents in the cytoplasm in an inactive form. Once induced by IL-1 $\beta$, NF- $\mathrm{kB}$ activation requires sequential phosphorylation, degradation of $\mathrm{IkBa}$, and NF- $\mathrm{kB}$ p65 translocates from the cytoplasm to the nucleus to induce the expression of inflammation-related genes, including TNF-a, MMPs, COX-2, PGE2, NO, iNOS, IL-6, and ADAMTS $[49,50]$. In addition to regulating TNF- $a$ and IL-1b expression, NF- $\mathrm{kB}$ is also activated by these cytokines [51], thus forming a vicious circle. In our study, immunohistochemistry and mRNA expression analyses revealed that NF- $\mathrm{KB}$ levels increased over time in the 
PFOA model of PI. Thus, persistent high expression of $\mathrm{NF}-\mathrm{KB}$ in the PFOA model may explain the persistent existence of developmental factors in articular cartilage. However, in this study,we did not investigate the phosphorylation of NF- $\mathrm{kB}$, so we only speculated that early patellofemoral articular cartilage degeneration in a rat model of PI is associated with activation of the NF- $\mathrm{kB}$ signaling pathway, further studies are needed to clarify how NF-kB contributes to PFOA pathology.

There are some limitations in our study. First, although this PFOA model cannot be translated to human $\mathrm{OA}$, it can be used to study the therapeutic effects of drugs on OA. Second, because the shape of the patellar in rats is relatively small and difficult to obtain, we have not studied the changes of patellar cartilage degeneration. Third, we need to further study at the cellular level the molecular mechanism of the NF- $\mathrm{kB}$ signaling pathway in patellofemoral cartilage degeneration.

\section{Conclusions}

A rat model of PI-induced PFOA was successfully established by studying changes in cartilage and subchondral bone. This animal model may be useful for future research on PFOA. In addition, the PI-induced degeneration of patellofemoral cartilage may be related to the activation of the NF- $\mathrm{kB}$ signaling pathway and may deteriorate with time.

\section{Abbreviations}

PI: Patellar instability; PFOA: Patellofemoral osteoarthritis; OA: Osteoarthritis; PFJ: Patellofemoral joint; MMP-13: Matrix metalloproteinase-13; MicroCT: Micro-computed tomographic; qRT-PCR: Quantitative real-time polymerase chain reaction; BV/TV: Bone volume to total volume; BMD: Bone minera density; TB: Trabecular; SP: Deparation; SMI: Dtructure model index; PF: Pattern factor; DA: Degree of anisotropy

\section{Acknowledgements}

The authors would like to thank the staff of all participating departments. We thank Eva Lasic, PhD, from Edanz Editing (www.liwenbianji.cn/ac), for editing the English text of a draft of this manuscript.

\section{Authors' contributions}

$W L, Y K D$, and FW designed the study and performed the surgery. JHN, ML, and $\mathrm{WL}$ performed the experiments. GMY and YKD evaluated the data. WL wrote the manuscript, and HJK, YZN made the figures and tables. All authors participated in the writing of the manuscript and agreed to the publication.

\section{Funding}

This work was supported by the National Natural Science Foundation of China (grant no. 81873983), Key Program of Natural Science Foundation of Hebei Province (grant no. H2019206694), Hebei Province Key Project of Achievement Transformation (grant no.zh2018007), and the Fund for Graduates' Innovative Projects of the Academic Degree Office of Hebei Provincial Department of Education (grant no. CXZZBS2020114).

\section{Availability of data and materials}

The detailed data and materials of this study were available from the corresponding author through emails on reasonable request.

\section{Ethics approval and consent to participate}

This study was approved by the Animal Ethics Committee of the Third Hospital of Hebei Medical University (approval number: Z2019-005-1).
Consent for publication

Not applicable.

\section{Competing interests}

The authors declare that they have no competing interests.

Received: 31 January 2020 Accepted: 11 January 2021

Published online: 18 January 2021

\section{References}

1. Murray CJ, Vos T, Memish ZA, et al. Disability-adjusted life years (DALYs) for 291 diseases and injuries in 21 regions, 1990-2010: A systematic analysis for the Global Burden of Disease Study 2010. Lancet. 2012; 380(9859): 2197 - 223.

2. Bei M, Tian F, Zhang L, et al. A Novel Rat Model of Patellofemoral Osteoarthritis Due to Patella Baja, or Low-Lying Patella. Med Sci Monit. 2019; 25:2702-2717.

3. Kim YM, Joo YB. Patellofemoral osteoarthritis. Knee Surg Relat Res. 2012; 24(4): 193-200.

4. Davies AP, Vince AS, Shepstone L, Donell ST, Glasgow MM. The radiologic prevalence of patellofemoral osteoarthritis. Clin Orthop Relat Res. 2002;402: 206-12.

5. Schiphof D, van Middelkoop M, Bierma-Zeinstra SM,et al. Crepitus is a first indication of patellofemoral osteoarthritis (and not of tibiofemoral osteoarthritis). Osteoarthritis Cartilage. 2014; 22(5):631-8.

6. Dejour H, Walch G, NoveJosserand L, Guier C. Factors of patellar instability: an anatomic radiographic study. Knee Surg Sports Traumatol Arthrosc. 1994; 2(1): 19-26.

7. Dietrich TJ, Fucentese SF, Pfirrmann CW. Imaging of individual anatomical risk factors for patellar instability. Semin Musculoskelet Radiol. 2016; 20(1):65-73.

8. Otsuki S, Okamoto Y, Neo M, et al. Patellofemoral reconstruction for patellar instability with patella alta in middle-aged patients: Clinical outcomes. Orthop Traumatol Surg Res. 2018;104(2):217-221.

9. Steensen RN, Bentley JC, Trinh TQ, Backes JR, Wiltfong RE. The prevalence and combined prevalences of anatomic factors associated with recurrent patellar dislocation: a magnetic resonance imaging study. Am J Sports Med. 2015;43(4): 921-927.

10. Huri G, Atay OA, Ergen B, Atesok K, Johnson DL, Doral MN. Development of femoral trochlear groove in growing rabbit after patellar instability. Knee Surg Sports Traumatol Arthrosc. 2012;20:232-8.

11. Fithian DC, Paxton EW, White $L M$, et al. Epidemiology and natural history of acute patellar dislocation. Am J Sports Med. 2004;32(5):1114-21.

12. Diederichs G, Issever AS, Scheffler S. MR imaging of patellar instability: injury patterns and assessment of risk factors. Radiographics. 2010; 30(4):961 - 81.

13. Amis AA, Firer P, Mountney J, Senavongse W, Thomas NP. Anatomy and biomechanics of the medial patellofemoral ligament. Knee. 2003;10(03):215 20.

14. Collins NJ, Hinman RS, Menz HB, Crossley KM: Immediate effects of foot orthoses on pain during functional tasks in people with patellofemoral osteoarthritis: A cross-over, proof-of-concept study. Knee. 2017:24(1):76-81.

15. Naruse K, Urabe K, Jiang SX, et al. Osteoarthritic changes of the patellofemoral joint in STR/OrtCrlj mice are the earliest detectable changes and may be caused by internal tibial torsion. Connect Tissue Res. 2009:50(4):243 - 55.

16. Takahashi I, Matsuzaki T, Kuroki H, Hoso M. Induction of osteoarthritis by injecting monosodium iodoacetate into the patellofemoral joint of an experimental rat model. PLoS One. 2018;13(4): e0196625.

17. Gerwin N, Bendele AM, Glasson S, Carlson CS. The OARSI histopathology initiative recommendations for histological assessments of osteoarthritis in the rat. Osteoarthritis Cartilage. 2010:18 Suppl 3:S24-34.

18. Clark AL, Leonard TR, Barclay LD, Matyas JR, Herzog W. Heterogeneity in patellofemoral cartilage adaptation to anterior cruciate ligament transection; Chondrocyte shape and deformation with compression. Osteoarthritis Cartilage. 2006;14(2):120 - 30.

19. Clark AL, Leonard TR, Barclay LD, Matyas JR, Herzog W. Opposing cartilages in the patellofemoral joint adapt differently to long-term cruciate deficiency: Chondrocyte deformation and reorientation with compression. Osteoarthritis Cartilage. 2005;13(12): 1100-14.

20. Chang NJ, Shie MY, Lee KW, Chou PH, Lin CC, Chu CJ. Can early rehabilitation prevent posttraumatic osteoarthritis in the patellofemoral joint after anterior cruciate ligament rupture? Understanding the pathological features. Int J Mol Sci. 2017;18(4):829 
21. Plaas A, Osborn B, Sandy JD, et al. Aggrecanolysis in human osteoarthritis: Confocal localization and biochemical characterization of ADAMTS5hyaluronan complexes in articular cartilages. Osteoarthritis Cartilage. 2007; 15(7):719 - 34

22. Chang JK, Chang LH, Ho ML, et al. Parathyroid hormone 1-34 inhibits terminal differentiation of human articular chondrocytes and osteoarthritis progression in rats. Arthritis Rheum. 2009;60(10):3049-60.

23. Orlandi A, Oliva F, Tarantino U, et al. Transglutaminase-2 differently regulates cartilage destruction and osteophyte formation in a surgical model of osteoarthritis. Amino Acids. 2009;36(4):755 - 63.

24. Amin AR. Regulation of tumor necrosis factor-alpha and tumor necrosis factor converting enzyme in human osteoarthritis. Osteoarthritis Cartilage.1999;7(4): 392-4.

25. Tak PP, Firestein GS. NF-kappaB: a key role in inflammatory diseases. J Clin Invest. 2001:107(1):7-11.

26. Kopaladze RA. Methods for the euthanasia of experimental animals-the ethics, esthetics and personnel safety. Usp Fiziol Nauk. 2000;31(3):79-90.

27. Zatroch KK, Knight CG, Reimer JN, Pang DS. Refinement of intraperitoneal injection of sodium pentobarbital for euthanasia in laboratory rats (Rattus norvegicus). BMC Vet Res. 2017;13(1):60

28. Guangmin Yang, Faquan Li, Fei Wang, et al. The dysplastic trochlear sulcus due to the insufficient patellar stress in growing rats. BMC Musculoskelet Disord. 2019;20(1):411.

29. Dai Y, LU J, Li F, Yang G, Ji G, Wang, F. Changes in cartilage and subchondral bone in a growing rabbit experimental model of developmental trochlear dysplasia of the knee. Connect Tissue Res. 2019, 12;1-14.

30. Aulin C, Lundback P, Palmblad K, Klareskog L, Erlandsson Harris H. An in vivo cross-linkable hyaluronan gel with inherent anti-inflammatory properties reduces $O A$ cartilage destruction in female mice subjected to cruciate ligament transection. Osteoarthritis Cartilage. 2017;25(1):157 - 65.

31. Pritzker KP, Gay S, van den Berg WB, et al. Osteoarthritis cartilage histopathology: grading and staging. Osteoarthritis Cartilage. 2006;14(1):13-29.

32. Ranstam J. Repeated measurements, bilateral observations and pseudoreplicates, why does it matter? Osteoarthritis Cartilage. 2012;20(6):473-5.

33. Wyndow N, Collins N, Vicenzino B, Tucker K, Crossley K. Is There a Biomechanical Link Between Patellofemoral Pain and Osteoarthritis? A Narrative Review. Sports Med. 2016; 46(12):1797 - 808.

34. Guevara JM, Moncayo MA, VacaGonzález JJ, Gutiérrez ML, Barrera LA, GarzónAlvarado, DA. Growth plate stress distribution implications during bone development: A simple framework computational approach. Comput Methods Programs Biomed. 2015; 118(1):59-68.

35. Zhen G, Wen $C_{1}$ Cao X, et al. Inhibition of TGF-beta signaling in mesenchymal stem cells of subchondral bone attenuates osteoarthritis. Nat Med. 2013;19(6): $704-12$.

36. Martel-Pelletier J, Barr AJ, Pelletier JP, et al. Osteoarthritis. Nat Rev Dis Primers. 2016; 2: 16072

37. Boileau C, Martel-Pelletier J, Fahmi H, Mineau F, Boily M, Pelletier JP. The peroxisome proliferator-activated receptor $y$ agonist pioglitazone reduces the development of cartilage lesions in an experimental dog model of osteoarthritis: In vivo protective effects mediated through the inhibition of key signaling and catabolic pathways. Arthritis Rheum. 2007; 56 (7):2288-98.

38. Malemud CJ, Islam N, Hagai TM: Pathophysiological mechanisms in osteoarthritis lead to novel therapeutic strategies. Cells Tissues Organs, 2003; 174: 34-48.

39. Schlomann U, Wildeboer D, Webster A, et al. The metalloprotease disintegrin ADAM8. Processing by autocatalysis is required for proteolytic activity and cell adhesion. J Biol Chem, 2002; 277: 48210-19.

40. DeFrate LE, Kim-Wang SY, Englander ZA, MCNulty AL: Osteoarthritis year in review 2018: Mechanics. Osteoarthritis Cartilage, 2019; 27(3): 392-400.

41. Chubinskaya S, Kuettner KE, Cole AA: Expression of matrix metalloproteinases in normal and damaged articular cartilage from human knee and ankle joints. Lab Invest, 1999; 79: 1669-77.

42. Glasson SS, Askew R, Morris EA, et al. Deletion of active ADAMTS5 prevents cartilage degradation in a murine model of osteoarthritis. Nature. 2005; 434(7033):644-8.

43. Hagiwara Y, Ando A, Chimoto E, Saijo Y, OhmoriMatsuda K, Itoi E. Changes of articular cartilage after immobilization in a rat knee contracture model. $J$ Orthop Res. 2009; 27:236-242.

44. Martel-Pelletier J, Boileau C, Pelletier JP, Roughley PJ. Cartilage in normal and osteoarthritis conditions. Best Pract Res Clin Rheumatol, 2008:22(2):351 - 84
45. Van der Kraan PM, van den Berg WB. Chondrocyte hypertrophy and osteoarthritis: role in initiation and progression of cartilage degeneration? Osteoarthritis Cartilage. 2012;20(3): 223 - 32.

46. Pelletier JP, Martel-Pelletier J, Abramson SB. Osteoarthritis. Arthritis Rheum. 2001;44(6):1237-47.

47. Huebner JL, Seifer DR, Kraus VB. A longitudinal analysis of serum cytokines in the Hartley guinea pig model of osteoarthritis. Osteoarthritis Cartilage. 2007;15(3):354-6.

48. Goldring SR, Goldring MB. The role of cytokines in cartilage matrix degeneration in osteoarthritis, Clin Orthop Relat Res. 2004; (427 Suppl) S2736.

49. Miagkovn AV, Kovalenko DV, Makarov SS, et al. NF-kappaB activation provides the potential link between inflammation and hyperplasia in the arthritic joint. Proc Natl Acad Sci USA.1998,95(23),13859-64.

50. Marcu KB, Otero M, Olivotto E, Borzi RM, Goldring MB. NF-kappaB signaling. Curr Drug Targets. 2010;11(5):599-613.

51. Fan Z, Bau B, Yang H, Aigner T. IL-1 beta induction of IL-6 and LIF in normal articular human chondrocytes involves the ERK. Cytokine. 2004;28(1):17-24.

\section{Publisher's Note}

Springer Nature remains neutral with regard to jurisdictional claims in published maps and institutional affiliations.
Ready to submit your research? Choose BMC and benefit from:

- fast, convenient online submission

- thorough peer review by experienced researchers in your field

- rapid publication on acceptance

- support for research data, including large and complex data types

- gold Open Access which fosters wider collaboration and increased citations

- maximum visibility for your research: over $100 \mathrm{M}$ website views per year

At $\mathrm{BMC}$, research is always in progress.

Learn more biomedcentral.com/submissions 